\title{
Felicitous Labellings of Some Network Models*
}

\author{
Jiajuan Zhang ${ }^{1}$, Bing Yao ${ }^{1}$, Zhiqian Wang ${ }^{2}$, Hongyu Wang ${ }^{1}$, Chao Yang', Sihua Yang ${ }^{1}$ \\ ${ }^{1}$ College of Mathematics and Statistics, Northwest Normal University, Lanzhou, 730070, China; ${ }^{2}$ School of Mathematics Physics and \\ Software Engineering, Lanzhou Jiaotong University, Lanzhou 730070, China. \\ Email: yy\}918@163.com
}

Received 2013

\begin{abstract}
Building up graph models to simulate scale-free networks is an important method since graphs have been used in researching scale-free networks. One use labelled graphs for distinguishing objects of communication and information networks. In this paper some methods are given for constructing larger felicitous graphs from smaller graphs having special felicitous labellings, and some network models are shown to be felicitous.
\end{abstract}

Keywords: Felicitous Labelling; Set-Ordered Felicitous Labelling; Symmetric Graphs;Trees

\section{Introduction}

Graphs have been used in researching scale-free networks ([4],[9]). Many graph models can be labelled for distinguishing objects in communication networks. Ichishma and Oshima [3] investigated the relationship between partitional graphs and strongly graceful graphs and partitional graphs and strongly felicitous graphs. Yao, Chen, Yao and Cheng [6] show several relationships among several well-known labellings including felicitous labelling. In [1], among the graphs known to be felicitous are: $C_{n}$ except when $n \equiv 2(\bmod 4) ; K_{m, n}$ when $m, n>1$; $P_{2} \cup C_{2 n+1} ; P_{2} \cup C_{2 n} ; P_{3} \cup C_{2 n+1} ; S_{m} \cup C_{2 n+1} ; K_{n}$ if and only if $n \leq 4 ; P_{n}+K_{m}$; the friendship graph $C_{n}^{(3)}$ for $\mathrm{n}$ odd; $P_{n} \cup C_{3} ; P_{n} \cup C_{n+3}$. It has been noticed that some felicitous graphs in literature can be constructed, such as some classes of felicitous trees are obtained in [5]. Graham and Sloane [2] conjectured: Every tree is felicitous. We will present several methods for constructing larger felicitous graphs from smaller graphs having special felicitous labellings, and show some network models to be felicitous, such as edge-symmetric and near-symmetric graphs that are related with some models of self-similar and hierarchical networks in current research of complex networks.

Standard terminology and notation of graph theory are used here. Simple graphs are finite, undirected, no multiple edges and loopless, unless otherwise specified. The shorthand notation $[m, n]$ stands for an integer set $\{m$, $m+1, \ldots, n\}$ with $n>m \geq 0$. A $(p, q)$-graph is a simple graph with $p$ vertices and $q$ edges. A proper labelling $f$ of a $(p, q)$-graph $G$ is a mapping from $V(G)$ to $[m, n]$ such that

*This research is supported by the National Natural Science Foundation of China (No. 61163054 and No. 61163037). $f(x) \neq f(y)$ for distinct $x, y \in V(G)$.

Definition 1 [1] Suppose that a $(p, q)$-graph $G$ has a proper labelling $f: V(G) \rightarrow[0, q]$. The edge label $f(u v)$ of each edge $u v \in E(G)$ is defined as $f(u v)=f(u)+f(v)(\bmod q)$. If the edge label set $\{f(u v): u v \in E(G)\}=[0, q-1]$, then we say both $G$ and $f$ to be felicitous.

For the purpose of simplicity, we write $f(S)=\{f(x)$ : $x \in S \subseteq V(G)\}$ and $f(E(G))=\{f(u v): u v \in E(G)\}=\{f(u)+f(v)$ $(\bmod q): u v \in E(G)\}$ and $f\langle G\rangle=\{f(u)+f(v): u v \in E(G)\}$ for a felicitous labelling $f$ of a $(p, q)$-graph $G$ throughout this paper. Very often, the labelling $h(x)=q-f(x)$ for each verte $x \in V(G)$ is called the dual labelling of the labelling $f$. The notation $S(\bmod q)$ stands for the set $\{x(\bmod q): x \in S\}$ for a set $S$ of non-negative integers. For a set $[6,14]=\{6,7,8,9,10,11,12,13,14\}$, as an example, [6,14] $(\bmod 10)=\{0,1,2,3,4,6,7,8,9\}$, and $[6,14](\operatorname{bmod} 7)=[0,7]$; for $S=\{3,7,9,10,12,13\}$, then $S(\bmod 9)=\{0,1,3,4,7\}$.

In [7] and [8], the authors introduced the set-ordered graceful labellings and the set-ordered odd-graceful labellings: Let $(X, Y)$ be the bipartition of a bipartite $(p, q)$-graph $G$. If $G$ admits a (an odd-)graceful labelling $\mathrm{f}$ such that $\max \{f(u): u \in X\}<\min \{f(v): v \in Y\}$, then we call $f$ a emphset-ordered (odd-)graceful labelling, and denote this case as $f(X)<f(Y)$. Motivated from the above set-ordered (odd-)graceful labellings we define a set-ordered felicitous labelling as follows.

Definition 2. Let $(X, Y)$ be the bipartition of a bipartite $(p, q)$-graph $G$. If $G$ admits a felicitous labelling $f$ such that $\max \{f(u): u \in X\}<\min \{f(v): v \in Y\}$, then we call $f$ a set-ordered felicitous labelling and $G$ a set-ordered felicitous graph, and write this case as $f(X)<f(Y)$, and $f$ is called an optimal set-ordered felicitous labelling if $f\langle G\rangle=[b, b+q-1]$ and $f\langle G\rangle(\bmod q)=[0, q-1]$. 
Let $G_{i}$ be the ith copy of a $(p, q)$-graph $G$ with $p \geq 3$ for $i \in[1, n], n \geq 2$. Every vertex $u^{0} \in V(G)$ has its corresponding vertices $u_{i}^{0} \in V\left(G_{i}\right)$ for $i \in[1, n]$. We have a so-called root graph $H_{0}$ on $n$ vertices, where $V\left(H_{0}\right)=\left\{v_{i}: i \in[1, n]\right\}$. The graph $H_{1}$ obtained by identifying one vertex $v_{j} \in V\left(H_{0}\right)$ with one $\mathrm{u}^{\wedge} 0 \mathrm{j} \in \mathrm{V}\left(G_{j}\right)$ for $j \in[1, n]$ is called an edge-symmetric graph, denoted as $H_{1}=\left\langle H_{0}, G\right\rangle$. Clearly, the graph $H_{1}-E\left(H_{0}\right)$ has n components that are isomorphic to $G$. From the definition of $H_{1}$, we can get the edge-symmetric graphs $H_{i}+1=\left\langle H_{i}, G\right\rangle$ for $i \in[1, N]$ such that each component of $H_{i}+1-E\left(H_{i}\right)$ is isomorphic to $G$. Let $T$ be a $(n, m)$-graph and let $G$ be a $(p, q)$-graph. We define a near-symmetric graph $H=\langle T \bullet G\rangle$ such that $H$ contains $T$ and $n$ edge-disjoint copies $G_{i}$ of $G$ with $|E(H-E(T))|=n q,|H| \leq n p$ and $i \in[1, n]$.

\section{Results}

Lemma 1. Suppose that a connected $(p, q)$-graph $G$ has a felicitous labelling $f$. Then

(i) $f\langle G\rangle=[\lambda, \lambda+q-1]$ and $f\langle G\rangle(\bmod q)=[0, q-1]$, where $\lambda=\min f\langle G\rangle$.

(ii) The dual labelling $g$ of $f$ is also felicitous, and $g(x y)=q-f(x y)$ for each edge $x y \in E(G)$ with $f(x y) \neq 0$. Furthermore, $f$ is (optimal) set-ordered felicitous when $G$ is bipartite, so is $g$.

Lemma 2. Suppose that a bipartite graph $G$ admits set-ordered felicitous labellings. Then each set-ordered felicitous labelling of $G$ satisfies that one vertex of $G$ is labelled by zero and another vertex of $G$ is labelled by the number of edges of $G$.

Lemma 3. Suppose that a bipartite $(p, q)$-graph $G$ is set-ordered felicitous, and $G^{\prime}$ is a copy of $G$. Joining a vertex $u \in V(G)$ with its corresponding vertex $u^{\prime} \in V\left(G^{\prime}\right)$ produces a set-ordered felicitous graph.

Theorem 4. Let $G_{1}, G_{2}$ be two disjoint bipartite graphs having optimal set-ordered felicitous labellings. Then there exist vertices $u \in V\left(G_{1}\right)$ and $v \in V\left(G_{2}\right)$ such that the graph obtained by joining $u$ with $v$ or by identifying $u$ with $v$ into one vertex is optimal set-ordered felicitous.

Theorem 5. Let $T$ be a set-ordered felicitous tree and let $G$ be a connected $(p, q)$-graph having optimal set-ordered felicitous labellings. The near-symmetric graph $\mathrm{H}=\langle T \bullet G\rangle$ is felicitous.

In general, a near-symmetric graph $\langle T \bullet G\rangle$ having felicitous labellings may not be set-ordered felicitous. We

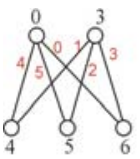

(a)

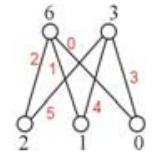

(b)

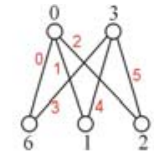

(c)

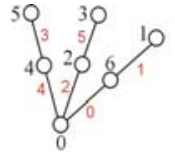

(d)

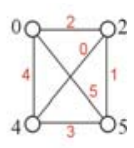

(e)
Figure 1. (a) An optimal set-ordered felicitous labelling $\boldsymbol{f}$ of $K_{2,3}$ with $f\left\langle K_{2,3}\right\rangle=[4,9]$; (b) the dual labelling of $f$; (c) a nonset-ordered felicitous labelling of $K_{2,3}$; (d) a non-set-ordered felicitous labelling of a 2-star; (e) a felicitous labelling of $K_{4}$. define the following matchable graphs and compound graphs. Let $T$ be a set-ordered felicitous tree on $2 n$ vertices. $T$ has a set-ordered felicitous labelling $f$ such that $f\left(x_{i}\right)=i-1$ for $i \in[1,2 n]$.

For each $k \in[1, n]$, there exists a graph $S k$ having $2 m$ vertices and $2 q$ edges with respect to integers $m, q \geq 1$ such that $V\left(S_{k}\right)=X_{k} \cup Y_{k}$, where $X_{k}=\left\{x_{k, j}: j \in[1, m]\right\}$ and $Y_{k}=\left\{y_{k, j}\right.$ : $j \in[1, m]\}$. Furthermore, $S_{k}$ is connected or has just two components if it is disconnected. If $S_{k}$ has just two components $S_{k, 1}$ and $S_{k, 2}$, then $x_{k, 1} \in V\left(S_{k, 1}\right)$ and $y_{k, 1} \in V\left(S_{k, 2}\right)$, and we call $x_{k, 1}, y_{k, 1}$ the bases of $S_{k}$, and $S_{k}$ is called $a$ matchable graph if there is a labelling $g$ such that $S_{k}$ satisfies the following:

(1) $g\left(x_{k, 1}\right)=\mathrm{k}(q+1)+f\left(x_{k}\right), k \in[1, n]$;

(2) $g\left(x_{k, j}\right)+\mathrm{g}\left(y_{k, j}\right)=M, j \in[1,2 m]$, where $M=2 n(q+1)-1$;

(3) $\mathrm{g}\left(S_{k}\right)=[M-k(q+1)+1, \quad M-k(q+1)+q] \cup[k(q+1)-q$, $k(q+1)-1]$.

Write $S_{k}=C\left(T ; 2 m, 2 q ; x_{k, 1}, y_{k, 1}\right), k \in[1, n]=[1,|T| / 2]$. Then we can construct a compound graph $T^{*}=\mathrm{C}\left[S_{1}, S_{2}\right.$, $\left.\ldots, S_{n} ; T\right]$ by identifying $x_{k, 1} \in V\left(S_{k}\right)$ with $x_{k} \in V(T)$, and $y_{k, 1} \in \mathrm{V}\left(S_{k}\right)$ with $x_{2 n-k+1} \in V(T)$ for $k \in[1, n]$; and by identifying those vertices with the same labels in $S_{2}, S_{4}, \ldots, S_{2 z}$, where $z=[n / 2]$. It follows Theorem 5 that every compound graph is felicitous.

Corollary 6. Every compound graph $T^{*}=\mathrm{C}\left[S_{1}, S_{2}, \ldots\right.$, $\left.S_{n} ; T\right]$ is felicitous.

Lemmas 2, 3 and Theorem 4 can be applied to construct matchable graphs by the way used in the proof of Theorem 5. It is noticeable, some compound graphs $T^{*}=C\left[S_{1}, S_{2}, \ldots, S_{n} ; T\right]$ having felicitous labellings are not bipartite. If trees $T$ holds $|T| \leq 8$, then a near-symmetric graph $\mathrm{H}=\langle T \bullet G\rangle$ is just an edge-symmetric graph $\mathrm{H}=\langle T$, $G\rangle$. Figueroa-Centeno, Ichishima, and Muntaner-Batle [1] define a felicitous graph to be strongly if there exists an integer $k$ such that every edge $u v$ of the graph holds

$$
\min \{f(u), f(v)\} \leq k<\max \{f(u), f(v)\} .
$$

Theorem 7. A connected graph $G$ admits a strongly felicitous labelling $h$ if and only if $h$ is a set-ordered felicitous labelling.

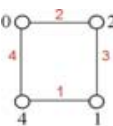

(a)

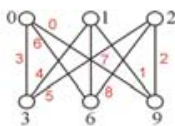

(b)

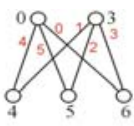

(c)

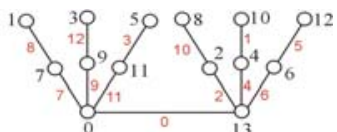

(d)
Figure 2. Four graphs having optimal set-ordered felicitous labellings. (a) $f\left\langle K_{2,3}\right\rangle=[2,5]$; (b) $f\left\langle K_{3,3}\right\rangle=[3,11]$; (c) $f\left\langle K_{2,3}\right\rangle=[4,9]$; (d) $f\langle T\rangle=[7,19]$.
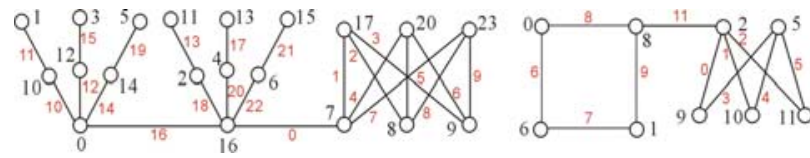

Figure 3. Based on the graphs shown in Figure 2, two optimal set-ordered felicitous graphs are used for illustrating Theorem 4. 


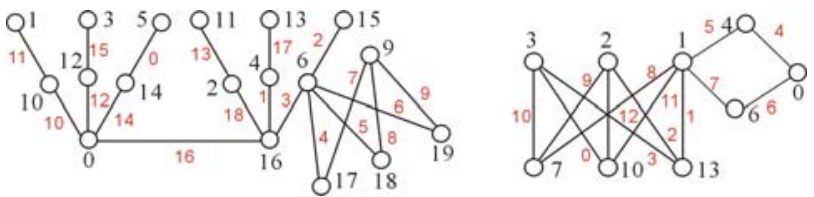

Figure 4. Based on the graphs shown in Figure 2, two optimal set-ordered felicitous graphs are used for illustrating Theorem 4.

\section{Conclusion}

It may be meaning to consider the following problems.

(1) Determine simple graphs having (optimal) set-ordered felicitous labellings.

(2) If a connected graph $G$ has a set-ordered felicitous labeling $\mathrm{f}$, then is $\mathrm{f}$ optimal?

(3) A simple graph $G$ has a felicitous labelling $f$. Do there exist edges $x y \in E(G)$ and $u v \in E\left(G^{c}\right)$ such that $\mathrm{f}$ is still a felicitous labelling of the graph $G-x y+u v$ ?

(4) Is a matchable graph felicitous?

Proof of Lemma 1. To show the assertion (i), we can see $f\langle G\rangle=E_{<q} \cup E_{\geq q}$, where $E_{<q}=\{f(x)+f(y)<q: \quad x y \in E(G)\}$

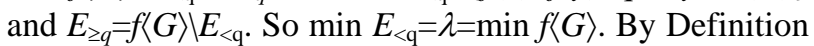
|refdefn:definition11, $f\langle G\rangle(\bmod q)=[0, q-1]$. On the other hand, $f(E(G))=E_{<q} \cup E_{\geq q}(\bmod q)=[0, q-1]$, which implies $f\langle G\rangle=[\lambda, \lambda+q-1]$. If $E_{\geq q}(\bmod q) \neq[0, \lambda-1]$, then $\mid E_{<q} \cup E_{\geq q}$ $(\bmod q) \mid \leq q-1$; a contradiction. So we have $E_{<\mathrm{q}}=[\lambda, q-1]$ and $E_{\geq q}(\bmod q)=[0, \lambda-1]$.

We show the assertion (ii). Notice that the dual labelling $g$ of $f$ is defined as $g(x)=q-f(x)$ for $x \in V(G)$. For every edge $x y \in E(G)$ we have $g(x)+g(y)+f(x)+f(y)=2 q$. Hence, $g(x y)+f(x y)=q$ for $f(x y) \neq 0$. Since $f(x)+f(y)=q$ if $f(x y)=0$, so $g(x)+g(y)=q$, which means $g(x y)=0(\bmod q)$. Therefore, $g(E(G))=[0, q-1]$ according to $f(E(G))=[0$, q-1]. For $x y \in E(G), f(x y)=f(x)+f(y)$ if $f(x)+f(y) \leq q-1$, we have $g(x)+g(y)=2 q-[f(x)+f(y)]=q+q-f(x y)$; and $f(x y)=$ $f(x)+f(y)-q$ if $f(x)+f(y)>q$, so

$g(x)+g(y)=2 q-[f(x)+f(y)]=2 q-[f(x)+f(y)-q+q]=q-f(x y)$.

Therefore, $g(x y)=g(x)+g(y) \quad(\bmod \quad q)=q-f(x y)$ for $x y \in E(G)$ and $f(x y) \neq 0$. If $G$ is bipartite, let $(X, Y)$ be the bipartition of $G$. Suppose that $\mathrm{f}$ is set-ordered felicitous, that is, $f(X)<f(Y)$. Clearly, $g(Y)<g(X)$. If $\mathrm{f}$ is optimal set-ordered felicitous, so $f\langle G\rangle=[b, b+q-1]$ with $b=\min f(Y)$. Hence, $g\langle G\rangle=[2 q-(b+q-1), 2 q-b]=[\mathrm{q}-b+1,2 q-b]$, however, $q-b+1=\min g(X)$. The proof of the assertion (ii) is over.

Proof of Lemma 2. Let $G$ be a bipartite connected $(p, q)$-graph that admits a set-ordered felicitous labelling g. For the bipartition $(X, Y)$ of $V(G)$, where $X=\left\{X_{i}\right.$ : $i \in[1, s]\}$ and $Y=\left\{y_{j}: j \in[1, t]\right\}$ with $s^{+} t=p$, without loss of generality, we let $g\left(x_{i}\right)<g\left(x_{i+1}\right)$ for $i \in[1, s-1], g\left(x_{s}\right)<g\left(y_{1}\right)$, and $g\left(y_{j}\right)<g\left(y_{j+1}\right)$ for $j \in[1, t-1]$ by the choice of the labelling $g$.

Assume that $g\left(x_{1}\right)>0$ and $g\left(y_{t}\right)<q$. Let $k_{0}=g\left(y_{1}\right)$. Since $g\left(x_{i}\right) \in\left[1, k_{0}-1\right]$ and $g\left(y_{j}\right) \in\left[k_{0}, q-1\right]$ for each edge $x_{i} y_{j} \in E(G)$, so we have $g\left(x_{i}\right)+g\left(y_{j}\right) \in\left[k_{0}+1, k_{0}+q-2\right]$. However, the set $\left[k_{0}+1, k_{0}+q-2\right]$ contains at most (q-2) numbers, so $\left[k_{0}+1, k_{0}+q-2\right](\bmod q)=[0, q-2]$, which is contrary with $[0, q-1]=g(E(G))$. If $g\left(x_{1}\right)=0$ and $g\left(y_{t}\right)<q$, then we have $\mathrm{g}\left(x_{i}\right) \in\left[\begin{array}{ll}0, & \left.k_{0}-1\right]\end{array}\right.$ and $\mathrm{g}\left(y_{j}\right) \in\left[\begin{array}{ll}k_{0}, & q-1]\end{array}\right.$ for $x_{i} y_{j} \in E(G)$, and furthermore $g\left(x_{i}\right)+g\left(y_{j}\right) \in\left[k_{0}+1, k_{0}+q-2\right]$. However, $\left[k_{0}+1, k_{0}+q-2\right](\bmod q)=\left[0, k_{0}-2\right] \cup\left[k_{0}, q-1\right]$ contradicts with the choice of the labelling $g$. The proof of the lemma is complete.

Proof of Lemma 3. Let $G$ be a $(p, q)$-graph described in the theorem's hypothesis, and let $(X, Y)$ be the bipartition of $V(G)$, where $X=\left\{x_{i}: i \in[1, s]\right\}$ and $Y=\left\{y_{j}: j \in[1, t]\right\}$ with $s+t=p$. $G$ admits a set-ordered felicitous labelling $g$ with $g\left(x_{i}\right)<g\left(x_{i+1}\right)$ for $i \in[1, s-1], \quad g\left(x_{s}\right)<g\left(y_{1}\right), \quad$ and $g\left(y_{j}\right)<g\left(y_{j+1}\right)$ for $j \in[1, t-1]$, and $g\left(x_{1}\right)=0$ and $g\left(y_{t}\right)=q$ by Lemma 2.

Let $G^{\prime}$ be a copy of $G$ with its bipartition $\left(X^{\prime}, Y^{\prime}\right)$, where $X^{\prime}=\left\{x_{i}^{\prime}: i \in[1, s]\right\}$ and $Y^{\prime}=\left\{y_{j}^{\prime}: j \in[1, t]\right\}$ are the copies of $X$ and $Y$, and let $g^{\prime}$ be a copy of $g$ with $g^{\prime}\left(x_{1}^{\prime}{ }_{1}\right)=0$ and $g\left(y_{t}^{\prime}\right)=q$. Joining the vertex $x_{1} \in X \subseteq V(G)$ with its corresponding vertex $x_{1}^{\prime} \in X^{\prime} \subseteq V\left(G^{\prime}\right)$ together by an edge produces a bipartite graph $H$ with its bipartition $\left(X_{H}, Y_{H}\right)$, where $X_{H}=X \cup Y^{\prime}$ and $Y_{H}=X^{\prime} \cup Y$. Let $a=g\left(y_{1}\right)=g^{\prime}\left(y_{1}^{\prime}\right)$. Based on two labellings $g$ and $g$ ' we define a labelling $f$ of $H$ as:

(1) $f(u)=g(u)$ for $u \in X$, $f(v)=a+q-g^{\prime}(v)$ for $v \in Y^{\prime}$;

(2) $f(y)=q-a+1+g(y), y \in Y ; f(x)=2 q+1-g^{\prime}(x), x \in X^{\prime}$.

Notice that $f(X) \subseteq[0, a-1]$, $f\left(Y^{\prime}\right)=\left\{a+q-g^{\prime}(u): u \in Y^{\prime}\right\} \subseteq[a, q]$,

$f(Y)=\{q-a+1+g(v): v \in Y\} \subseteq[q+1,2 q-a+1]$, and $f\left(X^{\prime}\right)=\{2 q$ $\left.+1-g^{\prime}(v): v \in X^{\prime}\right\} \subseteq[2 q-a+2,2 q+1]$. Hence, $f(V(H)) \subseteq[0$, $2 q+1]$. Therefore, $f$ is proper and holds $f\left(X_{H}\right)<f\left(Y_{H}\right)$. For each edge $x_{i} y_{j} \in E(G) \subseteq E(H)$, we have

$q+1 \leq f\left(x_{i}\right)+f\left(y_{j}\right)=q-a+1+g\left(x_{i}\right)+g\left(y_{j}\right) \leq 2 q$.

Correspondingly, for each edge $x_{i}^{\prime} y_{j}^{\prime} \in E\left(G^{\prime}\right) \subseteq E(H)$ which corresponds to the edge $x_{i} y_{j} \in E(G)$, $f\left(x_{i}^{\prime}\right)+f\left(y_{j}^{\prime}\right)=a+3 q+1-\quad\left[g^{\prime}\left(x_{i}^{\prime}\right)+g^{\prime}\left(y_{j}^{\prime}\right)\right]$, and furthermore $2 q^{\prime}+2 \leq f\left(x_{i}^{\prime}\right)+f\left(y_{j}^{\prime}\right) \leq 3 q+1$. Clearly, $f\left(x_{i}\right)+f\left(y_{j}\right)<f\left(x_{i}^{\prime}\right)+f\left(y_{j}^{\prime}\right)$ for each edge $x_{i} y_{j}$ and its corresponding edge $x_{i j}^{\prime} y_{j}^{\prime}$ in $H$. We obtain $f\langle G\rangle=[q+1,2 q]$ and $f\left\langle G^{\prime}\right\rangle=[2 q+2,3 q+1]$. Hence, $f(E(G))=[q+1,2 q], f\left(E\left(G^{\prime}\right)\right)=[1, q]$. Since

$f\left(x_{1}\right)+f\left(x_{1}^{\prime}\right)=g\left(x_{1}\right)+2 q+1-g^{\prime}\left(x_{1}^{\prime}\right)=2 q+1$,

so $f\left(x_{1} x_{1}^{\prime}\right)=f\left(x_{1}\right)+f\left(x_{1}^{\prime}\right)(\bmod 2 q+1)=0$. Thereby, $f$ is set-ordered felicitous because

$f(E(H))=f\langle G\rangle \cup\left\{f\left(x_{1} x^{\prime}{ }_{1}\right)\right\} \cup f\left\langle G^{\prime}\right\rangle(\bmod 2 q+1)=[0,2 q]$.

It is noticeable, $f\left(x_{i}\right)+f\left(x_{i}^{\prime}\right)=g\left(x_{i}\right)+2 q+1-g^{\prime}\left(x_{i}^{\prime}\right)=2 q+1$, which means $f\left(x_{i}\right)+f\left(x_{i}^{\prime}\right)(\bmod 2 q+1)=0$ for $x_{i} \in X$ and the corresponding vertex $x_{i}^{\prime} \in X^{\prime}$; and $f\left(y_{j}\right)+f\left(y_{j}^{\prime}\right)=q-a+1$ $+g\left(y_{j}\right)+a+q-g^{\prime}\left(y_{j}^{\prime}\right)=2 q+1, f\left(y_{j}\right)+f\left(y_{j}^{\prime}\right)(\bmod 2 q+1)=0$ for $y_{j} \in Y$ and the corresponding vertex $y_{j}^{\prime} \in Y^{\prime}$. So we can delete the edge $x_{1} x_{1}^{\prime}$ from $H$, and then join $x_{i} \neq x_{1}$ (resp. $y_{j}$ ) with its corresponding vertex $x_{i}^{\prime}$ (resp. $y_{j}^{\prime}$ ) by an edge together for $i \in[2, s]$ (resp. $j \in[1, t]$ ) such that the resulting 
graphs are set-ordered felicitous. The lemma is covered.

Proof of Lemma 4. Let $\left(X_{i}, Y_{i}\right)$ be the bipartition of vertices of a bipartite $\left(p_{i}, q_{i}\right)$-graph $G_{i}$ for $i=1,2$, where $X_{i}=\left\{x_{i, j}: j \in\left[1, s_{i}\right]\right\}$, and $Y_{i}=\left\{y_{i, j}: j \in\left[1, t_{i}\right]\right\}, s_{i}+t_{i}=p_{i}$. For $i=1,2$, let $f_{i}$ be an optimal set-ordered felicitous labelling of $G_{i}$ with $f_{i}\left(x_{i, j}\right)<f_{i}\left(x_{i, j+1}\right)$ for $\mathrm{j} \in\left[1, s_{i}-1\right], f_{i}\left(x_{i}, s_{i}\right)<f_{i}\left(y_{i, 1}\right)$, and $f_{i}\left(y_{i, j}\right)<f_{i}\left(y_{i, j+1}\right)$ for $j \in\left[1, t_{i}-1\right]$, and furthermore $f_{i}\left(x_{i, 1}\right)=0$ and $f_{i}\left(y_{i, t i}\right)=q_{i}$ according to Lemma 2. Thereby, we have

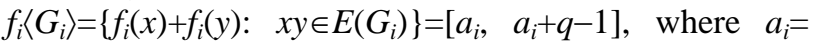
$f_{i}\left(y_{i, 1}\right), i=1,2$. Joining $y_{1, t 1} \in Y_{1}$ with $x_{2,1} \in X_{2}$ by an edge produces a new bipartite graph $H$ having the bipartition $\left(X_{1} \cup X_{2}, Y_{1} \cup Y_{2}\right)$. Clearly, $|V(H)|=p_{1}+p_{2},|E(H)|=q_{1}+q_{2}+1$. Let $M=q_{1}+q_{2}$. We extend the labellings $f_{1}, f_{2}$ to a labeling $f$ of $H$ in the following way:

(1) $f\left(x_{1, i}\right)=f_{1}\left(x_{1, i}\right)$ for $x_{1, i} \in X_{1}$ and $i \in\left[1, s_{1}\right]$;

(2) $f\left(x_{2,1}\right)=f_{2}\left(x_{2,1}\right)+a_{1}$ for $x_{2,1} \in X_{2}$ and $l \in\left[1, s_{2}\right]$;

(3) $f\left(y_{1, j}\right)=f_{1}\left(y_{1, j}\right)+a_{2}$ for $y_{1, j} \in Y_{1}$ and $j \in\left[1, t_{1}\right]$;

(4) $f\left(y_{2, k}\right)=f_{2}\left(y_{2, k}\right)+q_{1}+1$ for $y_{2, k} \in Y_{2}$ and $k \in\left[1, t_{2}\right]$.

Clearly, $f\left(X_{1}\right)=\left\{f_{1}\left(x_{1, i}\right): x_{1, i} \in X_{1}\right\} \subseteq\left[0, a_{1}-1\right]$,

$f\left(X_{2}\right)=\left\{f_{2}\left(x_{2,1}\right)+a_{1}: x_{2,1} \in X_{2}\right\} \subseteq\left[a_{1}, a_{1}+a_{2}-1\right]$,

$f\left(Y_{1}\right)=\left\{f_{1}\left(y_{1, j}\right)+a_{2}: y_{1, j} \in Y_{1}\right\} \subseteq\left[a_{1}+a_{2}, a_{2}+q_{1}\right]$, and

$f\left(Y_{2}\right)=\left\{f_{2}\left(y_{2, k}\right)+q_{1}+1: y_{2, k} \in Y_{2}\right\} \subseteq\left[a_{2}+q_{1}+1, M+1\right]$.

More details, $f\left(X_{1}\right)<f\left(X_{2}\right)<f\left(Y_{1}\right)<f\left(Y_{2}\right)$ in $H$, so $f\left(X_{1} \cup X_{2}\right)$ $<f\left(Y_{1} \cup Y_{2}\right)$ and $f(V(H)) \subseteq[0, M+1]$. We will show $f(E(H))=\{f(u)+f(v)(\bmod M+1): u v \in E(H)\}=[0, M]$. Notice that $f\left\langle G_{1}\right\rangle=\left\{f(u)+f(v): u v \in E\left(G_{1}\right) \subseteq E(H)\right\}=\left[a_{1}+a_{2}, a_{1}+a_{2}+q_{1}\right.$ $-1], f\left\langle G_{2}\right\rangle=\left\{f(x)+f(y): x y \in E\left(G_{2}\right)\right\}=\left[a_{1}+a_{2}+q_{1}+1, a_{1}+a_{2}+M\right]$,

$f\left(y_{1, t}\right)+f\left(x_{2,1}\right)=f_{1}\left(y_{1, t}\right)+a_{2}+f_{2}\left(x_{2,1}\right)+a_{1}=f_{1}\left(y_{1, t}\right)+a_{1}+a_{2}=q_{1}+a_{1}$ $+a_{2}$. Therefore, $f\langle H\rangle=\left[a_{1}+a_{2}, a_{1}+a_{2}+M\right]$ with $a_{1}+a_{2}=\min$ $f\left(Y_{1} \cup Y_{2}\right)$.

Case A1. $a_{1}+a_{2}<\mathrm{q} 2$. From $a_{1}+a_{2}<M+1$ and $a_{1}+a_{2}+q_{1}-1<\quad M+1$, so $f\left(E\left(G_{1}\right)\right)=f\left\langle G_{1}\right\rangle$. Since $f\left\langle G_{2}\right\rangle=\left[a_{1}+a_{2}+q_{1}+1, \quad M\right] \quad \cup\left[M+1, a_{1}+a_{2}+M\right]$, $f\left(E\left(G_{2}\right)\right)=\left[a_{1}+a_{2}+q_{1}+1, M\right] \cup\left[0, a_{1}+a_{2}-1\right]$. Thereby, we have

$f(E(H))=f\left(E\left(G_{1}\right)\right) \cup f\left(E\left(G_{2}\right)\right) \cup\left\{q_{1}+a_{1}+a_{2}\right\}=[0, M] . \quad$ (1)

Case A2. $a_{1}+a_{2}=q_{2}$. Then $f\left(E\left(G_{1}\right)\right)=\left[q_{2}, M-1\right], f\left(E\left(G_{2}\right)\right)$ $=\left[0, q_{2}-1\right]$. Hence, we obtain (1).

Case A3. $a_{1}+a_{2}=q_{2}+1$. For $f\left\langle G_{1}\right\rangle=\left[a_{1}+a_{2}, a_{1}+a_{2}+q_{1}-1\right]$ $=\left[q_{2}+1, M\right]$, and $f\left(E\left(G_{1}\right)\right)=\left[q_{2}+1, M\right]$. From $f\left\langle G_{2}\right\rangle=$ $\left[a_{1}+a_{2}+q_{1}+1, a_{1}+a_{2}+M\right]=\left[M+2, q_{2}+1+M\right]$, we have $f\left(E\left(G_{2}\right)\right)$ $=\left\{f(u)+f(v)(\bmod M+1): u v \in E\left(G_{2}\right)\right\}=\left[1, q_{2}\right]$. We obtain (1).
Case A4. $a_{1}+a_{2} \geq q_{2}+2$. Since $f\left\langle G_{1}\right\rangle=\left[a_{1}+a_{2}, M\right] \cup$ $\left[M+1, a_{1}+a_{2}+q_{1}-1\right]$, we have $f\left(E\left(G_{1}\right)\right)=\left[a_{1}+a_{2}, M\right] \cup[0$, $\left.a_{1}+a_{2}-q_{2}-2\right]$, and $f\left(E\left(G_{2}\right)\right)=\left[a_{1}+a_{2}-q_{2}, a_{1}+a_{2}-1\right]$, which means (1).

Based on the facts $f\left(X_{1}\right)<f\left(X_{2}\right)<f\left(Y_{1}\right)<f\left(Y_{2}\right), f\langle\mathrm{H}\rangle$ (mod $M+1)=[0, M]$ and $f(V(H)) \subseteq[0, M+1]$, and by the assertion (i) of Lemma 1 and by the definition of an optimal set-ordered felicitous labelling, we conclusion that $f$ is optimal set-ordered felicitous.

The proof of Lemma 4 is complete.

\section{REFERENCES}

[1] J. A. Gallian. A Dynamic Survey of Graph Labeling. The lectronic journal of combinatorics, 17 (2010), \# DS6.

[2] Graham R J, Sloane N J A. On additive bases and harmonious graphs. SIAM J Algebraic Discrete Mathods, 29(1) (1980), 382-404.

[3] Rikio Ichishma and Akito Oshima. On partitional and other related graphs. preprint.

[4] Li L., Alderson D., Tanaka R., Doyle J.C., and Willinger, W. Towards A Theory Of Scale-Free Graphs: Definition, Properties, And Implications. Internet Mathematics 2 (4) (2005), 431-523.

[5] Bing Yao, Ming Yao, Hui Cheng, Jin-wen Li, Ji-guo Xie and Zhong-fu Zhang. On Felicitous Labelling of Trees. The proceeding of The 4th International Workshop on Graph Labeling (IWOGL 2008), Harbin Engineering University and University of Ballarat, Australia, January, 2008. pp5-8.

[6] Bing Yao, Xiang'en Chen, Ming Yao, Hui Cheng. On ( $k$, ג)-magically total labeling of graphs. submitted to JCMCC.

[7] Bing Yao, Hui Cheng, Ming Yao and MeiMei Zhao. A Note on Strongly Graceful Trees. Ars Combinatoria 92 (2009), 155-169.

[8] Xiangqian Zhou, Bing Yao, Xiang'en Chen, and Haixia Tao. A proof to the odd-gracefulness of all lobsters. Ars Combinatoria, Volume CIII, January (2012),13-18.

[9] Bing Yao, Xiang'en Chen, Xiangqian Zhou,Jiajuan Zhang, Xiaomin Zhang, Ming Yao, Mogang Li, Jianming Xie. Graphs Related With Scale-free Networks. Proceeding of The 2nd International Conference on Electronics, Communications and Control (ICECC2012), October, 2012, Zhoushan, China, 284-287. 